\title{
Novel Metamaterial Structures with Low Loss at Millimeter Wave Frequency Range
}

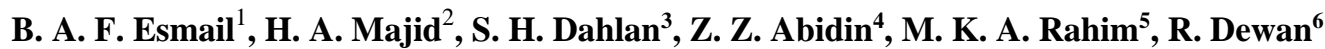 \\ ${ }^{1,2}$ Faculty of Engineering Technology, Universiti Tun Hussein Onn Malaysia, Parit Raja, Johor, Malaysia \\ ${ }^{3,4}$ Research Centre for Applied Electromagnetic, Universiti Tun Hussein Onn Malaysia, Parit Raja, Johor, Malaysia \\ ${ }^{5,6}$ Faculty of Electrical Engineering, Universiti Teknologi Malaysia, Johor, Malaysia
}

\begin{tabular}{|c|c|}
\hline Article Info & ABSTRACT \\
\hline Article history: & \multirow{10}{*}{$\begin{array}{l}\text { Two novel millimeter-wave (MMW) metamaterials (MTMs) unit cells operate } \\
\text { at } 28 \mathrm{GHz} \text { for a future fifth generation }(5 \mathrm{G}) \text { mobile network applications had } \\
\text { been designed, presented and numerically investigated. Two MTM structures } \\
\text { are proposed; namely the double E-shaped resonator (DER) and T-U shaped } \\
\text { resonator (TUSR). The DER and TUSR are consists of a double E and } \\
\text { combined T-U shaped, each printed on front side of the substrate layer } \\
\text { respectively. DER achieves a bandwidth of } 0.5 \mathrm{GHz} \text { and a return loss of }-34 \\
\mathrm{~dB} \text {, whereas TUSR features a bandwidth of } 0.3 \mathrm{GHz} \text { and a return loss of }-18 \\
\mathrm{~dB} \text {. The conventional split range resonator (SRR) was optimized to operate at } \\
\text { similar frequency for comparison purpose. The simulation results revealed that } \\
\text { the proposed DER and TUSR unit cells achieves the lowest loss as opposed to } \\
\text { literature with -0.09 dB ( } 0.99 \text { in linear scale) and - } 0.23 \mathrm{~dB} \text { ( } 0.97 \text { in linear scale) } \\
\text { respectively. Moreover, a well-known algorithm was used to extract the } \\
\text { constitutive parameters and the double negative nature of the two novel MTM } \\
\text { structures is proven. }\end{array}$} \\
\hline Received Nov 9, 2017 & \\
\hline Revised Jan 22, 2018 & \\
\hline Accepted Feb 21, 2018 & \\
\hline Keywords: & \\
\hline & \\
\hline MMW frequency range & \\
\hline MTMs losses & \\
\hline Negative refractive index & \\
\hline SRR & \\
\hline
\end{tabular}

Copyright $\odot 2018$ Institute of Advanced Engineering and Science. All rights reserved.

\section{Corresponding Author:}

Huda A. Majid

Faculty of Engineering Technology,

Universiti Tun Hussein Onn Malaysia,

86400 Parit Raja, Johor, Malaysia.

Email: mhuda@uthm.edu.my

\section{INTRODUCTION}

MTMs are artificial materials with unique responses to electromagnetic wave (EM). The MTMs are designed arbitrarily to realize many noteworthy properties such as the negative refractive index and the inverse Doppler shift due to the negative permittivity and permeability[1]. In 1967, these unnatural properties of the material were proven theoretically by Russian scientist Veselago and implemented in the experimentally stage by Pendry in 1998. [2,3]. Experimental results by Pendry depicting new phenomena and found numerous applications for these artificial materials such as the cloaking phenomena [4], perfect absorbers [5] and to enhance the performance and miniaturise the antenna [6-8]. In the literature, several techniques and processes had been usd to investigate and enhance the MTMs. However, the performance of the MTMs suffer from its narrow bandwidth and inherent MTMs losses that limits their spectrum applications and late enable MTMs based devices.

At a high-frequency range such as millimeter wave (MMW) band, MTMs experience high losses [9] whereas, the losses are very low and the unique electromagnetic properties of the MTMs can still be achieved within the microwave band. The losses gives negative influences and adverse effects toward the realizations of the extraordinary EM properties of the MTMs. Hence, the introduction of novel MTM structures with low loss is highly demanded to enable MTMs based devices, especially at the high-frequency range. 
In the literature, several possible strategies for overcoming the issue of high losses in MTMs had been proposed such as the tailoring geometry of MTMs unit cell [10,11], exhibit electromagnetically induced transparency (EIT) phenomena [12], using active devices [13]. On the other hand, the availability of wide bandwidth, high speed and high capacity of MMW band make it as a suitable choice for many applications such as in the gigabit wireless communications, imaging sensors, and deep space communications $[14,15]$. These advantages make this band as the best candidate for $5 \mathrm{G}$ cellular networks by exploiting the enormous amount of spectrum to greatly increase communication capacity[16].

In this paper, two novel MTMs structures are designed, investigated and simulated at $28 \mathrm{GHz}$. The Split Ring Resonator (SRR) unit cell is optimized at the same frequency to compare the results. The proposed DER and TUSR structures show a very high performance than the conventional SRR structure at the corresponding frequency regions by providing the highest transmission peak, $S_{21}$, as reported in the literature. Morever, the new structures can be used to tilt the main beam of the $5 \mathrm{G}$ antenna by loading the novel unit cells on the substrate of the antenna to produce different values of refractive index.

\section{RESEARCH METHOD}

\subsection{Design of The Novel MTM Structures}

The configuration of the DER and TUSR unit cells are shown in Figure 1(a) and (b). DER unit cell consists of double E-shaped at the front face of the structure. The T-shape and U-shape are combined to form TUSR unit cell. The inductance and capacitance effect has been introduced by the closed square loops and gaps of the two MTM structures, respectively. The resonant characteristic of the two MTM structures can be controlled by proper arrangement of these two paramters.

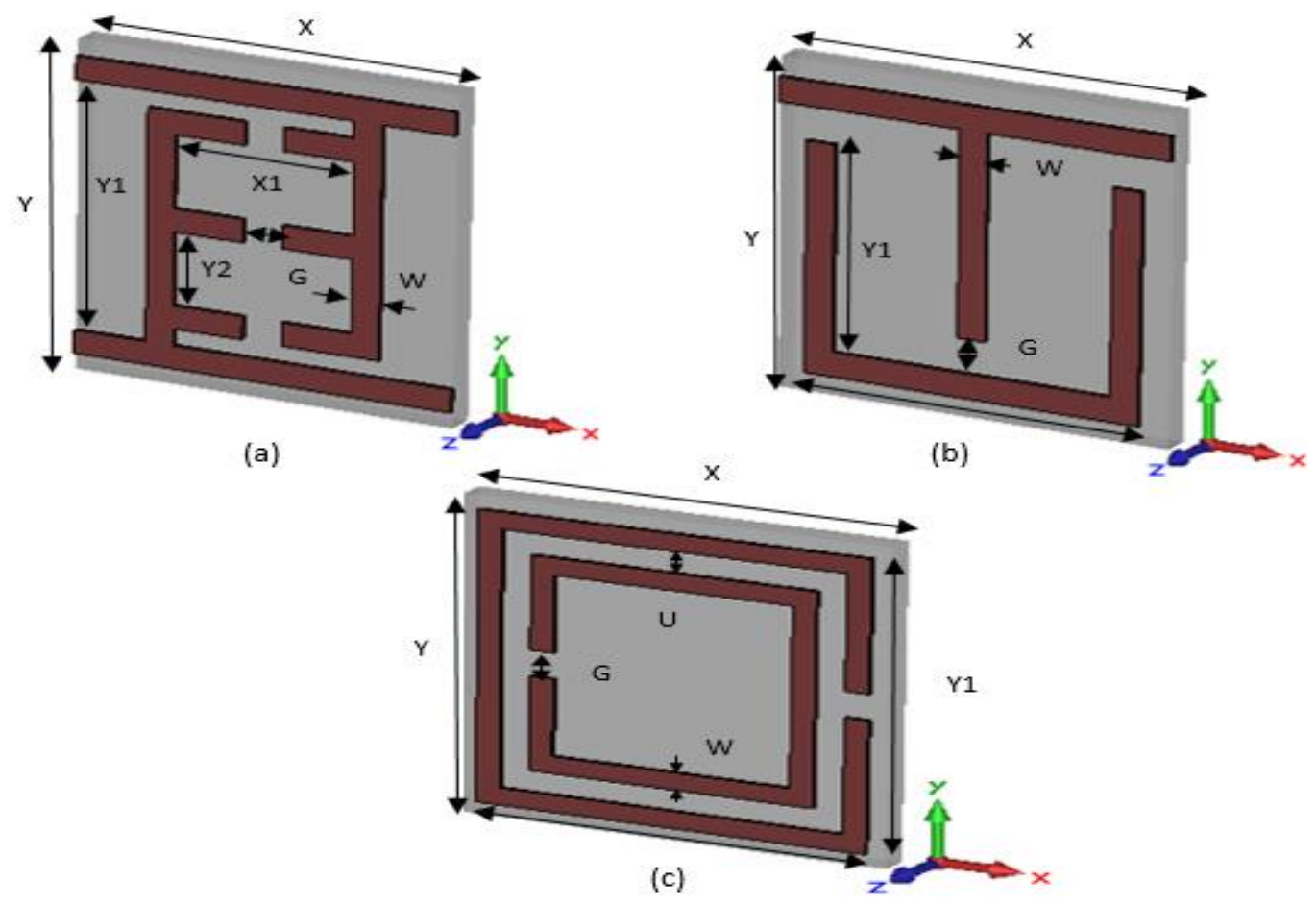

Figure 1. Metamaterial Structure of the a) Proposed DER Unit cell b) Proposed TUSR Unit Cell c) Conventional SRR Unit Cell with Dimensions of $\mathrm{X}=3.6, \mathrm{Y}=3.7, \mathrm{X} 1=3.3, \mathrm{Y} 1=3.4, \mathrm{U}=0.25, \mathrm{~W}=0.2$ and $\mathrm{G}=0.1$. All the Dimension in $\mathrm{mm}$.

For comparison purpose, SRR unit cell is introduced and depicted in Figure 1(c). The DER, TUSR and conventional SRR are constructed on the substrate (Rogers RT5880) with a thickness of $0.254 \mathrm{~mm}$, relative permittivity 2.2 and a tangent loss of 0.0009 . Table 1 shows the parameters dimensions of the two new designs and the optimized SRR dimensions are described in the caption of Figure 1(c). The lossy metal copper is the metallic layer of the three structures with the thickness of $0.035 \mathrm{~mm}$. The ports and boundary conditions are 
assigned to excite the EM wave and to extract the effective constitutive parameters of the proposed unitcells. The perfect magnetic conducting (PMC) and the perfect electric conducting (PEC) boundary conditions are applied along the $\mathrm{X}$ - and $\mathrm{Y}$-axis, respectively. The two waveguide ports are located in the $\mathrm{z}$-direction. The CST Microwave Studio based finite integration technique had been used to simulate the three MTM structures in the time domain solver.

Table 1. The DER and TUSR Dimensions at $28 \mathrm{GHz}$

\begin{tabular}{cccc}
\hline \multicolumn{2}{c}{ DER unit cell } & \multicolumn{2}{c}{ TUSR unit cell } \\
Parameter & Value $(\mathrm{mm})$ & Parameter & Value $(\mathrm{mm})$ \\
\hline X & 3.4 & $\mathrm{X}$ & 3.4 \\
Y & 3.5 & Y & 3.5 \\
Y1 & 2.6 & X1 & 3 \\
Y2 & 0.77 & Y1 & 2.1 \\
X1 & 1.6 & W & 0.25 \\
W & 0.25 & G & 0.35 \\
G & 0.35 & & \\
\hline
\end{tabular}

\section{NUMERICAL RESULTS AND DISCUSSION}

\subsection{Return Loss}

The implementation of the two new MTM structures and the conventional SRR unit cell that operate at MMW frequency range is described in this section in terms of the reflection coefficient. The two new structures are proposed to operate at $28 \mathrm{GHz}$ band which is the candidate band for $5 \mathrm{G}$ technology. The conventional SRR is optimized to operate at the same frequency for the comparison purpose. The return loss, $S_{11}$ of the DER and TUSR and SRR unit cells is revealed in Figure 2. It can be seen that the DER unit cell achieves the best reflection coefficient and bandwidth over other unit cells where the reflection coefficient was $-34 \mathrm{~dB}$ with a bandwidth of $0.5 \mathrm{GHz}$ at $28 \mathrm{GHz}$. On the other hand, the proposed TUSR and the conventional SRR unit cells introduce a bandwidth of $0.3 \mathrm{GHz}$ and $0.14 \mathrm{GHz}$, respectively. In comparison with the conventional SRR, our proposed structures present better results despite the common drawback of the narrow bandwidth of MTMs.

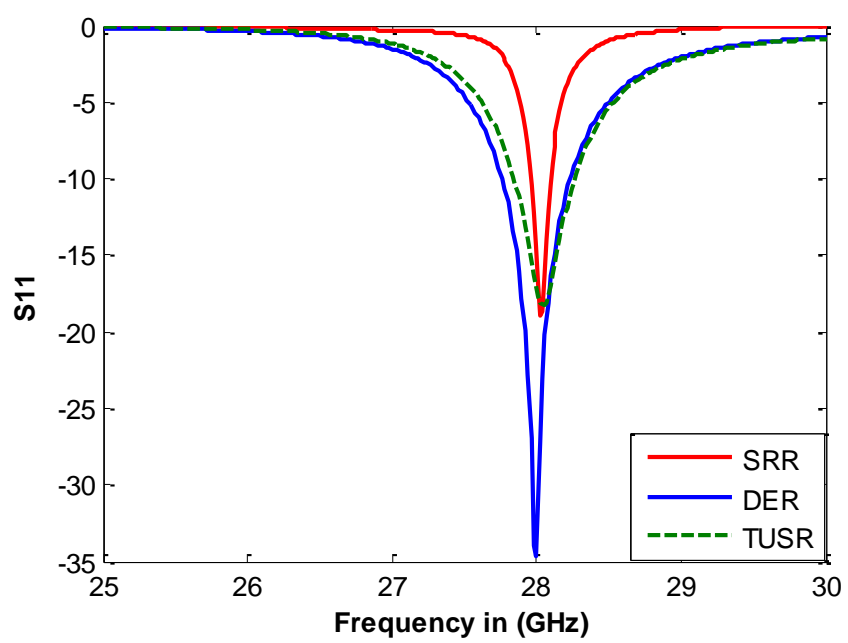

Figure 2. Reflection Coefficients, $S_{11}$ of the Proposed DER and TUSR and Conventional SRR Unit Cells

\subsection{Low Loss MTMs}

The MTM losses is a great challenge in the real environment due to its applications limitations, especially at high frequency range. The losses in MTMs at MMW frequency range are a big issue and the seeking for a low-loss structure at this range is highly demanded to enable the MTMs based devices. In this subsection, the losses of the proposed and the conventional MTM structures at $28 \mathrm{GHz}$ are analyzed and numerically investigated. The insertion loss $\left(S_{21}\right)$ is used to measure the losses of the MTM structures. For low loss, the near zero $(0) \mathrm{dB}$ in (dB scale) or near one (1) in (linear scale) of the transmission peak is highly 
demanded at the desired frequency. Figure 3 displays the transmission coefficients, $S_{21}$, of the proposed DER and TUSR unit cells and the conventional SRR structure.

As can be seen from Figure 3, the loss in the proposed DER unit cell is relatively smaller due to the proper geometrical arrangement where it achieves $-0.09 \mathrm{~dB}$ (0.99 in linear scale). This shows that the proposed unit cell achieves nearly the full transmission at the resonant frequency, $28 \mathrm{GHz}$. Otherwise, the TUSR unit cell introduces a loss of $-0.23 \mathrm{~dB}$ (0.97 in linear scale). The DER and TUSR unit cells present the lowest loss in comparison with the recent literature [17]. However, the highest loss is produced by the conventional SRR which is approximately $-1 \mathrm{~dB}$ (0.9 in linear scale).

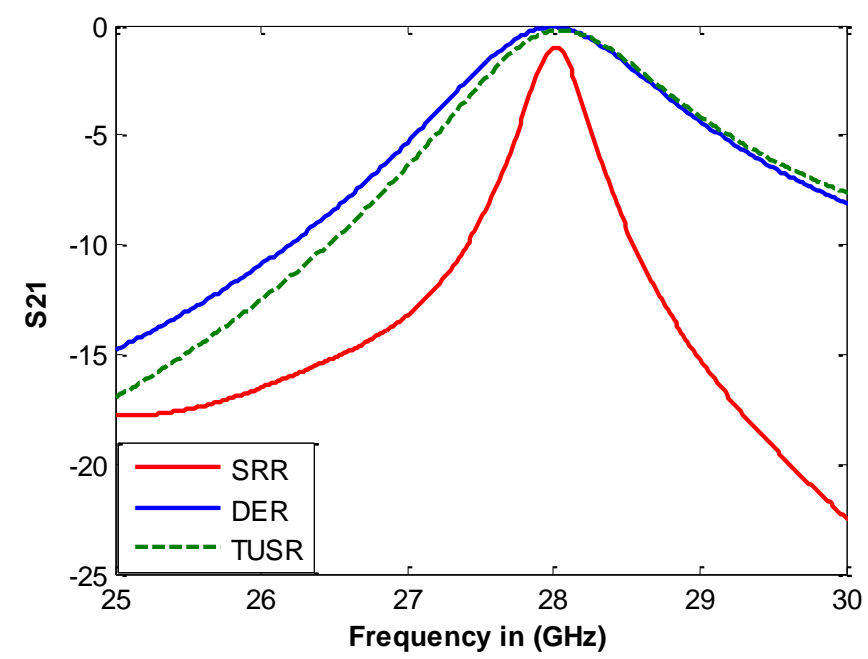

Figure 3. Transmission Coefficients, $S_{21}$ of Proposed DER and TUSR and Conventional SRR Unit Cells

The constitutive parameters are retrieved using the robust method as described in [18]. In this method, the effective refractive index $n$ and the impedance $z$ are first obtained from the extracted complex reflection and transmission coefficients as follows:

$$
\begin{aligned}
& Z=\sqrt{\frac{\left(1+S_{11}\right)^{2}-S_{21}^{2}}{\left(1-S_{11}\right)^{2}-S_{21}{ }^{2}}} \\
& e^{i n k d}=\frac{S_{21}}{1-S_{11} \frac{z-1}{z+1}}
\end{aligned}
$$

The refractive index is given by

$$
n=\frac{1}{k d}\left[\left\{\operatorname{Img}\left[\ln \left(e^{i n k_{o} d}\right)\right]+2 m \pi\right\}-i\left[\operatorname{Re}\left(\ln \left(e^{i n k_{o} d}\right)\right)\right]\right]
$$

Where $k_{o}$ the wave number and $d$ is is the thickness of the MTM structure. $n$ is the refractive index; $\mathrm{z}$ is the impedance and $m$ is the branch of the sinusoidal function periodicity. Then the permittivity and permeability can be calculated as follows:

$$
\begin{aligned}
& \varepsilon=\frac{n}{z} \\
& \mu=\mathrm{n} \times \mathrm{z}
\end{aligned}
$$

Figure 4 depicts the real parts of the refractive index, $n$ of the proposed DER, TUSR, and conventional SRR unit cells. The double negative nature is verified here by the negative refractive index, $n$ at the $28 \mathrm{GHz}$ for all three-unit cells. The negative $n$ is the most interesting classes of MTMs due to the possibility of 
diffraction unlimited imaging. We discuss the negative refractive index in the range of 25 to $30 \mathrm{GHz}$ as displayed in Figure 4. It is observed that the TUSR unit cell presents two regions of negative $n$ below and above the $28 \mathrm{GHz}$ while the DER structure displays region of negative $n$ above the resonant frequency. On the other hand, the negative $n$ area is above the $28 \mathrm{GHz}$ for SRR unit cell.

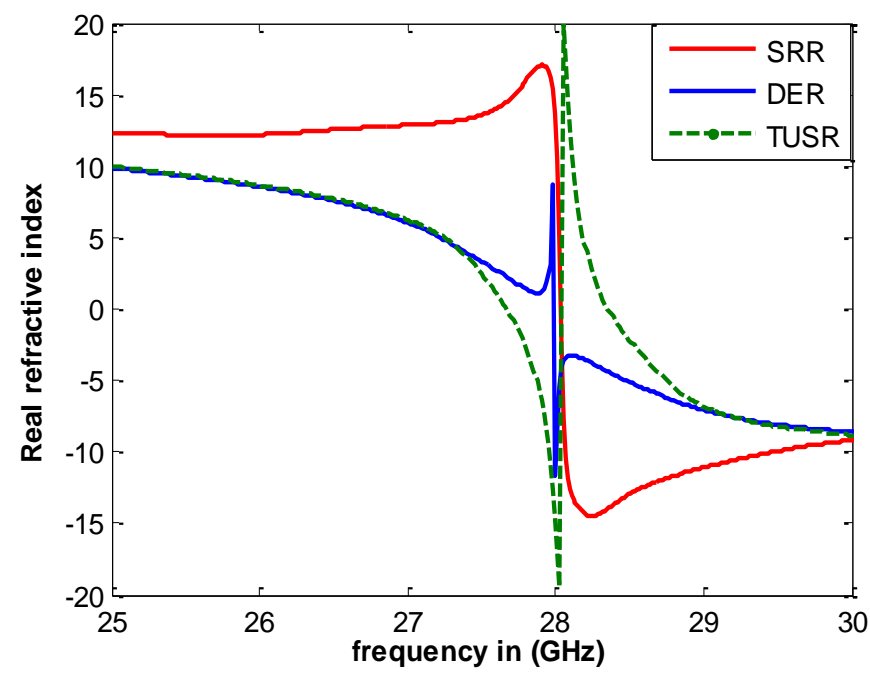

Figure 4. Real Parts Refractive Index of Proposed DER and TUSR and Conventional SRR Unit Cells

\section{CONCLUSION}

In conclusion, two novel MTM structures which are DER and TUSR are designed and numerically investigated at $28 \mathrm{GHz}$ which is the candidate band for $5 \mathrm{G}$ mobile network applications. The SRR unit cell is optimized to operate at the same frequency for comparison purpose. The DER, TUSR and SRR cells present bandwidth of $0.5 \mathrm{GHz}, 0.3 \mathrm{GHz}$ and $0.14 \mathrm{GHz}$, respectively. Moreover, DER and TUSR provide low loss by $-0.09 \mathrm{~dB}$ (0.99 in linear scale) and $-0.23 \mathrm{~dB}(0.97$ in linear scale), respectively in comparison with SRR where the loss is $-1 \mathrm{~dB}(0.9$ in linear scale). In addition, the double negative nature of the two novel MTM structures and conventional SRR unit cell is proven using a well-known algorithm where the real parts of the $n$ are negative at the resonant frequency. The proposed unit cells can be loaded to the $5 \mathrm{G}$ antenna to steer the main beam.

\section{ACKNOWLEDGEMENTS}

This work was supported by Ministry of Higher Education (MOHE), Faculty of Engineering Technology, Research Center of Applied Electromagnetics, Universiti Tun Hussein Onn Malaysia (UTHM) under Grant (Vote No: GPPS U735 / FRGS 1614).

\section{REFERENCES}

[1] Y Fan, T Qiao, F Zhang, Q Fu, J Dong, B Kong, H Li. "An electromagnetic modulator based on electrically controllable metamaterial analogue to electromagnetically induced transparency". Scientific reports. 2017;7.

[2] VG Veselago. "The electrodynamics of substances with simultaneously negative values of and $\mu$ ". Soviet physics uspekhi.vol. 1968; 10(4): 509.

[3] JB Pendry, et al. "Low frequency plasmons in thin-wire structures. Journal of Physics: Condensed Matter. 1998; 10(22): 4785.

[4] RK Dash, SK Sahu, CS Mishra, K Sethi, G Palai, S Sahu. "Realization of 'non-linear invisibility cloak'using metamaterial”. Optik-International Journal for Light and Electron Optics. 2016; 31; 127(20): 9635-9.

[5] Zhu J, D Li, S Yan, Y Cai, QH Liu, T Lin. "Tunable microwave metamaterial absorbers using varactor-loaded split loops". EPL (Europhysics Letters). 2015; 21; 112(5): 54002.

[6] PK Singhal, B Garg. "Design and Characterization of Compact Microstrip Patch Antenna Using" Split Ring" Shaped Metamaterial Structure“. International Journal of Electrical \& Computer Engineering (IJECE). 2012; 1; 2(5). 
[7] R Rajni, G Kaur, A "Marwaha. Metamaterial Inspired Patch Antenna for ISM Band by Adding Single-Layer Complementary Split Ring Resonators." International Journal of Electrical and Computer Engineering (IJECE). 2015; 1 ; 5(6): 1328-35.

[8] A Dadgarpour, B Zarghooni, BS Virdee, TA Denidni. "Improvement of gain and elevation tilt angle using metamaterial loading for millimeter-wave applications". IEEE Antennas and Wireless Propagation Letters. 2016; 15: 418-20.

[9] KL Tsakmakidis, MS Wartak, JJ Cook, JM Hamm, O Hess. "Negative-permeability electromagnetically induced transparent and magnetically active metamaterials. ", Physical Review B. 27;81(19):195128, May 2010.

[10] Y. Fan, Z. Wei, H. Li, H. Chen, CM .Soukoulis. "Low-loss and high-Q planar metamaterial with toroidal moment". Physical Review B. 2013; 15; 87(11): 115417.

[11] BAF Esmail, HA Majid, ZZ Abidin, SH Dahlan, MKA Rahim. "Reconfigurable Metamaterial Structure at Millimeter Wave Frequency Range". International Journal of Electrical and Computer Engineering (IJECE). 2017; 7(6): 29422949.

[12] Z Wei, X Li, N Zhong, X Tan, X Zhang, H Liu, H Meng, R Liang. "Analogue electromagnetically induced transparency based on low-loss metamaterial and its application in nanosensor and slow-light device". Plasmonics. 2016; 11; 3(12): 641-7.

[13] AD Boardman, VV Grimalsky, YS Kivshar, SV Koshevaya, M Lapine, NM Litchinitser, VN Malnev, M Noginov, YG Rapoport, VM Shalaev. "Active and tunable metamaterials". Laser \& Photonics Reviews. 2011; 8; 5(2): 287307.

[14] P Wang, Y Li, L Song, B Vucetic. "Multi-gigabit millimeter wave wireless communications for 5G: From fixed access to cellular networks". IEEE Communications Magazine. 2015; 53(1): 168-78.

[15] A Elboushi, A Sebak. "MMW sensor for hidden targets detection and warning based on reflection/scattering approach". IEEE Transactions on Antennas and Propagation. 2014; 62(9): 4890-4.

[16] Y Niu, Y Li, D Jin, L Su, AV Vasilakos. "A survey of millimeter wave (mmWave) communications for 5G: Opportunities and challenges". Wireless Networks. 2015; 1; 21(8): 2657-76.

[17] He Z, Geng Y. A wideband low-loss and small size left-handed metamaterial. In Communication Technology (ICCT), 2015 IEEE 16th International Conference on 2015; 18; 667-669. IEEE.

[18] X Chen, TM Grzegorczyk, BI Wu, Jr J Pacheco, JA Kong. "Robust method to retrieve the constitutive effective parameters of metamaterials". Physical Review E. 2004; 26; 70(1): 016608.

\section{BIOGRAPHIES OF AUTHORS}

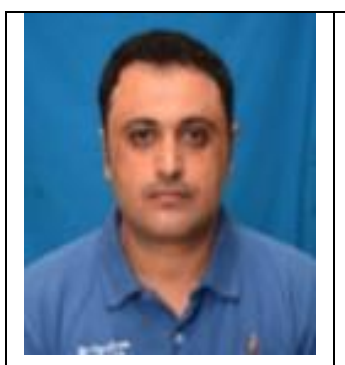

Bashar Ali Esmail received the B Eng. degree in Electrical Engineering (Telecommunications) with honours from Ibb University-Yemen, in 2008. He then obtained his M.Eng (Electrical Engineering) in 2016, at Universiti Tun Hussein Onn Malaysia. He is currently a on going $\mathrm{PhD}$ in Electrical Engineering at Electrical Engineering Technolgy Department, Faculty of Engineering Technolgy Universiti Tun Hussein Onn Malaysia. His research interest includes the areas of design of metamaterials structure, milimter wave antenna and pattern reconfigurable metamaterials antenna.

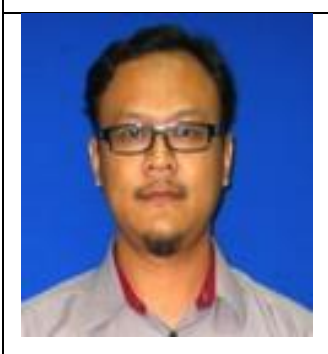

Huda A Majid received the B Eng. degree in Electrical Engineering (Telecommunication) from Universiti Teknologi Malaysia, in 2007. He then obtained his M.Eng in 2010 and PhD degrees in Electrical Engineering in 2013, at Universiti Teknologi Malaysia. He is currently a lecturer in the Department of Electrical Engineering Technology, Faculty of Engineering Technology, Universiti Tun Hussein Onn Malaysia. His research interest includes the areas of design of microstrip antennas, small antennas, Reconfigurable antennas, metamaterials structure, metalaterial antennas and millimeter wave antennas. He has published over 50 articles in journals and conference papers.

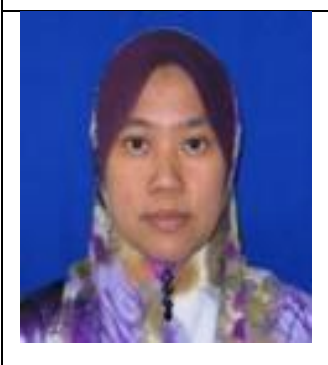

Zuhairiah Zainal Abidin was born in Kuala Lumpur, Malaysia, in 1978. She received the B.Eng.from the Universiti Teknologi Malaysia, in 2001, the M. Eng from the Kolej Universiti Tun Hussein Onn Malaysia, Johor, Malaysia, in 2003, and Ph.D. degree from Bradford University, U.K in 2011. Currently, she was a Senior Lecturer at Universiti Tun Hussein Onn Malaysia. Her current research interests include MIMO antenna design, electromagnetic bandgap (EBG) for wireless and mobile systems and high speed digital circuits and wearable antennas. 


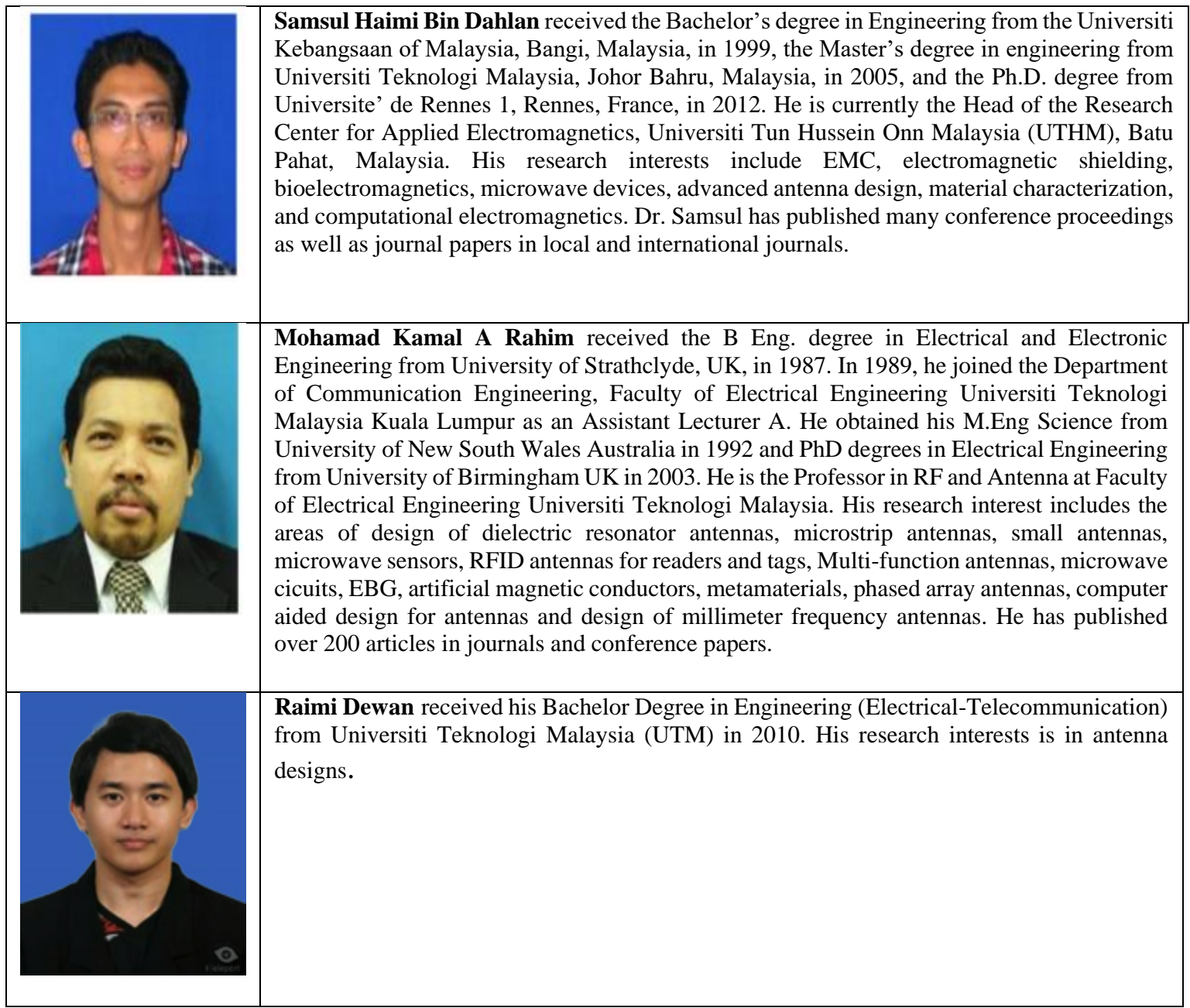

